\title{
Prevalencia de Chlamydia trachomatis y Neisseria gonorrhoeae en adolescentes chilenas
}

\author{
Andrea H uneeus ${ }^{1}$, María Gabriela Pumarino ${ }^{2}$, \\ Andrea Schilling ${ }^{1}$, Paz Robledo ${ }^{3}$, Magdalena Bofil ${ }^{4}$. \\ Rates of Chlamydia trachomatis \\ and Neisseria gonorrhoeae \\ in Chilean adolescents
}

Background: Chlamydia trachomatis and Neisseria gonorrhoeae are common sexually transmitted diseases among young women and little has been done to study them in Chilean adolescents. Aim: To determine the rates of $\mathrm{C}$ trachomatis and $\mathrm{N}$ gonorrhoeae in Chilean young women. Material and methods: Urine samples were obtained from 203 sexually active females under 25 year-old, who attended hospitals from either high or low income areas in Chile's capital, Santiago. C trachomatis and N gonorrhoeae were detected by nucleic acid amplification testing. Results: Seven percent of samples were positive for $\mathrm{C}$ trachomatis. All samples were negative for N gonorrhoeae. Among pregnant women, 19\% of samples were positive for $\mathrm{C}$ trachomatis, while non pregnant women were positive on $5.5 \%$ ( $\mathrm{p}$ $=0.04$ ). Systematic use of barrier contraception was referred by $12 \%$ of women. All of the latter were free of $\mathrm{C}$ trachomatis. No association was observed between age, number of sexual partners, age of first sexual intercourse, presence of urogynecological symptoms, socioeconomic status and the rate of $\mathrm{C}$ trachomatis. Conclusions: Seven percent of this group of Chilean young women was infected with C trachomatis. The figure rises to $19 \%$ if pregnant. Surveillance and screening programs should be implemented to prevent sequels on this vulnerable population (Rev Méd Chile 2009; 137: 1569-74).

(Key words: Chlamidia trachomatis; Coitus; Neisseria gonorrhoeae; Urine microbiology)

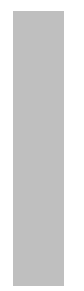

\begin{abstract}
Recibido el 23 de marzo, 2009. Aceptado el 27 de octubre, 2009.
Financiamiento: Roche Diagnostics Chile entregó los kits del Test Amplicore. Laboratorio Saval entregó los tratamientos para los pacientes positivos. Estos laboratorios no tuvieron relación con el diseño del protocolo. No hubo otras fuentes de financiamiento. No hay conflicto de intereses. ${ }^{1}$ Unidad de Atención Integral del Adolescente, Departamento de Obstetricia y Ginecolo-

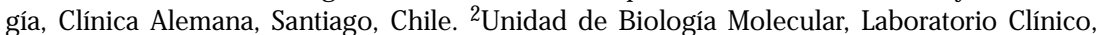
Clínica Alemana, Santiago, Chile. ${ }^{3}$ Departamento de Pediatría y de ${ }^{4}$ Ginecología y Obstetricia, Hospital Padre Hurtado, Santiago, Chile.
\end{abstract}

L a infección por Chlamydia trachomatis es la infección de transmisión sexual (ITS) de etiología bacteriana más frecuentemente reportada en

Correspondencia a: Dra. Andrea Huneeus. Manquehue 1410 piso 14, Vitacura, Santiago de Chile. Fono-Fax: 5622101210. E mail: ahuneeus@alemana.cl adolescentes. Estudios poblacionales han mostrado una prevalencia de $2,6 \%$ a $4,74 \%^{1,2}$. En estudios clínicos, las tasas comunicadas son aún más altas $5 \%$ a $15 \%{ }^{3-6}$. La infección por Neisseria gonorrhoeae es la segunda ITS más frecuente sujeta a notificación en Estados Unidos de Norteamérica. Estudios poblacionales han mostrado una prevalencia de gonorrea de $0,08 \%$ a $0,42 \% 1,2$. 
Las infecciones por $\mathrm{C}$ trachomatis y Ngonorrhoeae pueden producir complicaciones ginecológicas; como enfermedad inflamatoria pélvica (EIP) que se asocia a infertilidad tubaria, embarazo ectópico y dolor pélvico crónico; y complicaciones obstétricas y neonatales ${ }^{7}$. Estas infecciones aumentan la susceptibilidad para la transmisión del virus de inmunodeficiencia humana ${ }^{8}$.

Las ITS son un problema mayor para la salud de los jóvenes ya que casi la mitad de éstas ocumen entre los 15 y 24 años $^{9}$ y el mayor riesgo de adquirirlas es durante los primeros seis meses desde el inicio de la actividad sexual ${ }^{10}$. El impacto actual de estas infecciones en este grupo puede deberse a cambios en el comportamiento sexual; como la disminución de la edad de inicio de vida sexual, el número creciente de parejas sexuales, el uso inconsistente de preservativos, y a las dificultades específicas de la edad para acceder a servicios de salud apropiados.

Entre 50\% y $80 \%$ de los portadores de C trachomatis y N gonorrhoeae no refiere sintomatología uroginecológica. Esto hace difícil su diagnóstico precoz. Sin embargo, la detección temprana es de mayor importancia, porque estas infecciones son fácilmente tratables con dosis únicas de antimicrobianos que previenen las secuelas y la diseminación a otras parejas sexuales ${ }^{10}$.

En Chile no existen programas para el tamizaje ni monitoreo de prevalencia de $\mathrm{C}$ trachomatis. Autores locales han publicado tasas de positividad variable entre los diferentes grupos estudiados: $5,4 \%-6 \%$ en embarazadas $^{11,12}, 15,6 \%$ en trabajadoras sexuales ${ }^{13}$, 28,3\% en pacientes hospitalizadas por $\operatorname{EIP}^{14}, 5,7 \%$ en varones asintomáticos ${ }^{15}$, 24\% en mujeres que asisten a un consultorio de planificación familiar ${ }^{16}, 4,7 \%$ en mujeres de la región metropolitana ${ }^{17}$ y $3 \%$ en niñas prepuberales con vulvovaginitis con relato de abuso ${ }^{18}$. La gonorrea tiene un programa de monitoreo regulado por el Ministerio de Salud que ha mostrado una disminución progresiva de su tasa desde 1981 (113 por 100.000 habitantes) a 2006 (6,7 por $100.000)^{19}$. Un estudio local muestra $\mathrm{N}$ gonorrhoeae en $23,9 \%$ de las EIP ${ }^{14}$.

El gran porcentaje de enfermedad que cursa en forma asintomática ha hecho que las organizaciones de salud pública norteamericanas y europeas recomienden el tamizaje de $\mathrm{C}$ trachomatis anual en todos los menores de 25 años y en personas mayores con factores de riesgo. El tamizaje de gonorrea se recomienda para la población de riesgo $3,4,20$.
Hasta hoy, los test diagnósticos de ITS usados en Chile como la inmunofluorescencia directa (IFD) para $\mathrm{C}$ trachomatis y el cultivo de agar Thayer Martin para $\mathrm{N}$ gonorrhoeae, requerían una muestra de microorganismos vivos que necesariamente se debía obtener con un examen con espéculo. Con las técnicas modernas de amplificación de ácidos nucleicos se puede identificar el material genético de $C$ trachomatis y N gonorrhoeae en orina, autohisopado vaginal realizado por la paciente, muestras de Papanicolau líquido, hisopados cervicales y uretrales. Esta flexibilidad es una gran ventaja para adolescentes que temen el examen pélvico y para varones. También el almacenamiento y transporte de las muestras es más simple, ya que a $5^{\circ} \mathrm{C}$ se pueden conservar hasta 60 días sin afectar sus resultados. En C trachomatis, la detección de antígeno con IFD tiene una sensibilidad de $80 \%-85 \%$, los cultivos celulares, si bien son altamente específicos, tienen una sensibilidad cencana entre $70 \%$ y $85 \%$. Actualmente, la técnica de referencia o patrón de oro, es la amplificación de ácidos nucleicos, que detecta 20\%-30\% más muestras positivas que tecnologías previas con valores de sensibilidad y de especificidad de hasta $100 \%$ y $99,7 \%$, respectivamente ${ }^{21,22}$.

Como C trachomatis es un problema de salud pública escasamente estudiado en Chile, su impacto en el grupo de mayor riesgo, los menores de 25 años, se desconoce. Nuestro objetivo fue determinar prevalencia de Chlamydia trachomatis y Neisseria gonorrhoeae en adolescentes y jóvenes chilenas sexualmente activas. Secundariamente describimos características demográficas, hábitos sexuales y su relación con la presencia de infección.

\section{MateRIAL Y MÉTODO}

Registro prospectivo de cohorte de mujeres jóvenes y adolescentes sexualmente activas. La Organización Mundial de la Salud define a la gente joven al rango etario de 19 a 24 años y adolescentes al rango entre 10 y 18 años. Las pacientes de nivel socioeconómico medio-alto fueron enroladas de la Unidad de Adolescencia de Clínica Alemana de Santiago (CAS) y las de nivel socioeconómico bajo, en la Unidad de Adolescencia del Servicio de Pediatría y Maternidad del Hospital Padre Hurtado (HPH). Fueron incorporadas consecutivamente al estudio entre noviembre de 2006 y julio 2007. 
Se excluyeron quienes habían recibido antibióticos en el mes previo. Después de obtener el consentimiento de la paciente o del apoderado si ella era menor de 18 años, el médico tratante le practicó una entrevista confidencial estructurada. Se tomó muestra de orina de primer chorro que fue codificada y refrigerada a $5^{\circ} \mathrm{C}$ hasta su procesamiento en el Laboratorio de Biología Molecular de Clínica Alemana.

Las muestras fueron procesadas con reacción de polimerasa en cadena (PCR) usando el kitAmplicore ${ }^{\mathrm{TM}}$ de CT/NG-Roche ${ }^{\circledR}$. Cada etapa de la técnica se realizó en piezas separadas con equipamientos propios y flujo unidireccional. La preparación, extracción del ADN, amplificación, uso de controles intemos y la detección de los productos amplificados por medio de enzimoinmunoanálisis (ELISA), se realizaron siguiendo estrictamente las instrucciones del proveedor. Se hicieron controles negativos y positivos en cada serie, se usaron controles intemos para cada muestra que se coamplificaron simultáneamente para detectar inhibiciones de la amplificación. Las muestras inhibidas fueron repetidas en otro proceso. Para la amplificación de ADN se emplearon como partidores de C trachomatis CP24 y CP27 que define un segmento de 207 nucleótidos del plasmidio críptico y para N gonorrhoeae se usaron los partidores SSO1 SS02 que define una secuencia de 201 nucleótidos del gen de la citosina ADN metiltransferasa de NG. Ambos pares de partidores han sido ampliamente evaluados y la técnica está estandarizada en el kit comencial. El termociclador usado fue un PE Applied Biosystem GeneAmp PCR System 2400 Thermal Cycler. La detección del producto amplificado se realizó por medio de hibridización con sondas específicas. Los partidores tienen biotina, la que luego se revela en un ELSA con avidina peroxidasa. Este sistema de detección incrementa significativamente la sensibilidad de la técnica. El kit contiene enzima
AmpErase ${ }^{\circledR}$ para la eliminación de posibles segmentos ADN contaminantes (amplicones) previo a la reacción.

Los resultados positivos se comunicaban al médico tratante junto a la norma de tratamiento $\mathrm{y}$ seguimiento y los antibióticos para tratar a la paciente y sus contactos.

Este estudio fue revisado y aprobado por los Comités de Ética de Clínica Alemana de Santiago y Servicio de Salud Metropolitano Sur.

El número de pacientes fue calculado proyectando una prevalencia de $9 \%$ de $\mathrm{C}$ trachomatis en un universo de 2.000.000 de adolescentes chilenas de 15 a 24 años, con un intervalo de confianza de $95 \%$ y un error máximo de 4 . Esto da un tamaño muestral representativo mínimo de 197 pacientes.

Las comparaciones se hicieron usando el test exacto de Fisher o chi cuadrado para variables categónicas. Las variables continuas se analizaron con ANOVA (Pearson). Se consideró significación estadística con un intervalo de confianza de 95\% ( $\mathrm{p} \varangle 0,05)$. El programa estadístico utilizado fue Stadistical Packet for Social Science versión 16.0; ILL (Chicago).

\section{RESULTADOS}

Se enrolaron 203 pacientes con edades con rango entre 12 y 25 años, mediana de 18,7 y desviación típica de 3,127. La mediana de edad de primera relación sexual fue de 16,06 y del número de parejas sexuales fue 2,06 (rango 1 a 15). Los principales motivos de consulta fueron anticoncepción 44,3\% y embarazo $21,2 \%$. Ciento cuatro pacientes pertenecían al grupo de nivel socioeconómico medio-alto y 99 al de nivel socioeconómico bajo. Las de nivel medio-alto eran de mayor edad, tuvieron una mayor edad de inicio de vida sexual, y un mayor número de parejas sexuales que las de nivel socioeconómico bajo (Tabla 1).

\section{Tabla 1. Caracterización de la población estudiada total y por nivel socioeconómico: M edio-alto, Clínica Alemana de Santiago (CAS) y bajo, H ospital Padre H urtado (H PH )}

\begin{tabular}{|lcccc|}
\hline & Total & $\begin{array}{c}\text { Nivel socioeconómico } \\
\text { medio-alto }\end{array}$ & $\begin{array}{c}\text { Nivel socioeconómico } \\
\text { bajo }\end{array}$ & P \\
\hline Edad (años) & 18,7 & 20,69 & 16,1 & $<0,01$ \\
Edad inicio vida sexual & 16,06 & 17,82 & 14,25 & $<0,01$ \\
$\mathrm{~N}^{\circ}$ de parejas sexuales & 2,06 & 2,7 & 1,4 & $<0,01$ \\
Motivo consulta \#1 & Anticoncepción & Anticoncepción & Embarazo & $<0,01$ \\
& $44,3 \%(90)$ & $76,9 \%(80)$ & $42,4 \%(42)$ & \\
\hline
\end{tabular}


El 6,9\% de las pacientes fue positiva para C trachomatis y no hubo casos positivos de N gonorrhoeae. No hubo diferencias significativas entre las pacientes positivas y negativas para $\mathrm{C}$ trachomatis en edad $(17,79$ vs $18,77 \mathrm{p}=0,258)$, número de parejas sexuales $(1,36$ vs $2,12 \mathrm{p}=0,168)$ y edad de la primera relación sexual (16,06 vs 16,07 p =0,984). En el grupo de pacientes de nivel socioeconómico bajo hubo $10,1 \%$ de positividad para $C$ trachomatis y en el grupo de nivel socioeconómico medio-alto la positividad fue de 3,8\%. Esta diferencia tampoco fue estadísticamente significativa (Fisher $=0,068$ ).

Las pacientes embarazadas tuvieron una tasa de $19 \%$ de $C$ trachomatis significativamente mayor de que la tasa de las no embarazadas de 5,5\% (Fischer $=0,043$ ). Otros motivos de consulta (anticoncepción, trastornos menstruales, síntomas génito-urinarios, salud mental o abuso sexual) no se correlacionaron con mayor presencia de $\mathrm{C}$ trachomatis excepto embarazo (Tabla 2).

Frente a las preguntas dirigidas sobre síntomas uroginecológicos como disuria, flujo, prurito, goteo intermenstrual y algia pélvica, las pacientes portadoras de $\mathrm{C}$ trachomatis los relataban en 2,2\% $y$ las sanas en $8,3 \%$ ( $p=1,131)$.

El 12,3\% del total de las pacientes refirió usar preservativos siempre y ninguna de éstas estaba infectada C trachomatis. La tasa de infección de las usuarias consistentes de preservativo fue de $0 \%$ y en las usuarias no consistentes (uso ocasional o no uso) fue de 8,8\% ( $\mathrm{p}=0,141 \mathrm{~ns}$ ).

\section{CONCLUSIONES}

Se encontró una tasa de infección de $\mathrm{C}$ trachomatis de 6,9\% en adolescentes y jóvenes chilenas. Es urgente abordar las infecciones por Chlamydia en Chile de la misma forma en que se está haciendo en el resto del mundo $3,4,20$. Dado que la prevalencia en esta población fue alta, se hace innecesario confirmar los resultados ${ }^{22}$.

No se encontró gonorrea. Esto es concordante con los datos locales que muestran un progresivo descenso de las tasas ${ }^{19}$. Debido a la baja prevalencia esperada, se requerińa una muestra mayor para analizar esta patología.

La menor edad, menor edad de inicio de vida sexual y el mayor número de parejas sexuales no fueron factores de riesgo para $\mathrm{C}$ trachomatis en el grupo estudiado. Esto difiere de otras publicaciones que sí aparecen como factores de riesgo de infección en sexualmente activas ${ }^{1-6,9,10}$. La positividad de C trachomatis de las pacientes de nivel socioeconómico bajo fue más del doble que la positividad del nivel socioeconómico medio-alto. Sin embargo, estadísticamente esta diferencia no alcanzó a ser significativa. En la literatura, la prevalencia de $\mathrm{C}$ trachomatis ha demostrado tener diferencias relacionadas a grupos étnicos y factores socioculturales ${ }^{9,23}$.

Las pacientes embarazadas tuvieron más $C$ trachomatis que las no embarazadas. Esta tendencia en esta población vulnerable ha sido previamente reportada ${ }^{9}$ y su causa puede estar asociada a los comportamientos de riesgo que se concentran en las embarazadas jóvenes. La infección por Chlamydia aumenta las posibilidades de tener parto de pretérmino y rotura prematura de membranas y se ha asociado a un mayor riesgo de aborto espontáneo, bajo peso de nacimiento, mortalidad perinatal y endometritis postparto. La infección puede adquirirse por el feto como infección intrauterina o más frecuentemente a través de su paso por el canal del parto y esto resultar en una conjuntivitis neonatal o

Tabla 2. Relación de motivo de consulta con presencia de C trachomatis

\begin{tabular}{|lrccc|}
\hline M otivo de Consulta & $\mathbf{n}$ & \% C trachomatis & $\begin{array}{c}\text { \% C trachomatis en } \\
\text { reclutadas sin motivo } \\
\text { de consulta }\end{array}$ & $\mathbf{p}$ \\
\hline Anticoncepción & \multicolumn{3}{c|}{} & $0,350 \mathrm{~ns}$ \\
Embarazo & 90 & 5,6 & 5,0 & 0,043 \\
Trastornos menstruales & 43 & 19 & 8,2 & $0,076 \mathrm{~ns}$ \\
Síntomas génito-urinarios & 33 & 0,0 & 8,3 & $0,131 \mathrm{~ns}$ \\
Salud mental & 17 & 2,2 & 6,7 & $0,519 \mathrm{~ns}$ \\
Abuso sexual & 10 & 10,0 & 7,0 & $0,75 \mathrm{~ns}$ \\
\hline
\end{tabular}


neumonía ${ }^{24}$. Si la positividad de $\mathrm{C}$ trachomatis de este estudio se extrapola a los 90.112 recién nacidos chilenos que nacen cada año de madres menores de 25 años $^{25}$, habría 17.121 infecciones por $\mathrm{C}$ trachomatis anuales no diagnosticadas en este grupo. En Chile se ha encontrado $\mathrm{C}$ trachomatis en $8 \%$ de las conjuntivitis neonatales clínicas $^{26}$ y $18,1 \%$ de las neumonías del primer trimestre de vida ${ }^{27}$.

En este estudio la presencia de síntomas uroginecológicos no predijo la ocurrencia de infección. Este hallazgo se repite en la literatura ${ }^{10}$ que tampoco ha encontrado correlación de la infección con hallazgos clínicos del examen ginecológico como flujo mucopurulento, cervix friable, con ectropión o sensible ${ }^{28}$. La infección por Chlamydia se conoce como la "enfermedad silenciosa" porque la mayoría de las mujeres infectadas son asintomáticas ${ }^{7}$. La inconsistencia de la sintomatología unida a la alta prevalencia y los riesgos asociados a la infección han convertido al tamizaje anual de todas las jóvenes sexualmente activas en una medida más costo efectiva para controlar la enfermedad que otras intervenciones como el tamizaje de población sintomática ${ }^{29}$.

La mejor estrategia para prevenir infecciones de transmisión sexual es el uso de métodos de barrera $^{10}$, y ésta fue efectiva en todas nuestras pacientes que declararon usar condones siempre, ya que ninguna de ellas tuvo $\mathrm{C}$ trachomatis. En Chile, 42,6\% de los adolescentes refieren uso de preservativos la última vez que tuvieron actividad sexual $^{30}$ y en Estados Unidos de Norteamérica esta cifra en 2007 asciende a 61,5\%31. Se requiere hacer esfuerzos para mejorar la educación sexual y el acceso a preservativos a jóvenes para observar un uso más consistente.

\section{REFERENCIAS}

1. Miuer W, Ford C, Morris M, Handcock MS, Schmitz JL, HobBs MM Eт AL. Prevalence of chlamydial and gonococcal infections among young adults in the United States. JAMA 2004; 291: 2229-36.

2. Parish $\mathrm{W}$, Laumann E, Cohen M, Pan S, Zheng $\mathrm{H}$, Hoffman I ET AL. Population-based study of chlamydial infection in China: a hidden epidemic. JAMA 2003; 289: 1265-73.

3. La Montagne DS, Fenton KA, Randall S, Anderson S, CARTER P. Establishing the National Chlamydia Scree-
Este estudio tiene limitaciones. Primero el número de pacientes limitó el poder de la muestra para detectar diferencias significativas en la prevalencia de $\mathrm{C}$ trachomatis según características demográficas y hábitos sexuales. Segundo, su poder de generalización ya que se hizo sólo en dos locaciones urbanas. Se debe tener precaución al extrapolar sus resultados a otras poblaciones. Un estudio similar con una muestra mayor y más representativa del país puede resolver esto.

Este estudio tiene varias fortalezas. Primero fue prospectivo, segundo fue la muestra chilena más grande que se ha realizado en adolescentes y jóvenes. Tercero se usó amplificación de ácidos nucleicos en orina, una técnica sensible, específica, y con alta aceptabilidad entre adolescentes. Como esta técnica no está implementada masivamente en Chile, todavía es de muy alto costo. Sin embargo, los kits de amplificación de ácidos nucleicos modernos bajan significativamente sus precios al aumentar el número de muestras procesadas.

Con los resultados de este estudio y los que lo seguirán, es esperable que surja la necesidad de implementar programas de tamizaje masivo para jóvenes chilenos y que Chlamydia trachomatis sea incluida entre las enfermedades de notificación obligatoria. La primera fase de un programa de tamizaje debería implementarse en el control prenatal de mujeres menores de 25 años debido a su mayor tasa de enfermedad y los riesgos perinatales y neonatales asociados.

\section{Agradecimientos}

A Iris Delgado, del Departamento de Desamollo Médico de Clínica Alemana de Santiago por el análisis estadístico, y a la Dra. Francisca Ugarte por su colaboración en la edición.

ning Programme in England: results from the first full year of screening. Sex Transm Infect 2004; 80: 335-41.

4. ReKart ML, Brunham RC. Epidemiology of chlamydial infection: are we losing ground? Sex Transm Infect 2008; 84: 87-91.

5. Porras C, Safaeian M, González P, Hiddesheim A, Silva S, SChIFFMAN M ET AL. Epidemiology of Genital Chlamydia trachomatis Infection Among Young Women in Costa Rica. Sex Transm Dis 2008; 35: 461-8.

6. Farinati A, Zitto T, Bottiglieri R, Gastadello R, Cuffini C, CANNISTRACi R ET AL. Infecciones asintomáticas por Chlamydia trachomatis: un problema controlable 
en la población adolescente. Rev Panam Infectol 2008; 10: 8-12.

7. Anderson J. Sexually Transmitted Infections. En: Carpenter S, Rock J: Pediatric and Adolescent Gynecology. Philadelphia, Lippinccott Williams 2000; 293331.

8. Fleming D, Wasserheit J. From epidemiological synergy to public health policy and practice: the contribution of other sexually transmitted diseases to sexual transmission of HIV infection. Sex Transm Infect 1999; 75: 3-17.

9. Joesoef R, Satterwhite C, Weinstock H, Bradiey S, Braxton J, Clanton S et al. 2006 Chlamydia Prevalence Monitoring Project Annual Report. Sex Transm Dis Surv 2006; 9-18. Disponible en www.cdc.gov (Consultado 6 de septiembre de 2009).

10. Emans J. Sexually Transmitted Infections. En Emans: Pediatric and Adolescent Gynecology. Philadelphia, Lippincott Williams \& Wilkins 2005; 565-614.

11. Aliaga P, Bernal J, Martínez A, Dabancens A, Fernández $\mathrm{P}$, NAZER J. Incidencia de Chlamydia trachomatis en el embarazo. Rev Chil Obstet Ginecol 1985; 50: 140-9.

12. Bernal J, Martínez A, Triantafilo J Suárez M, DabanCens A, Hurtado C et al. Diagnóstico de enfermedades de transmisión sexual en adolescentes embarazadas chilenas. Rev Chil Obstet Ginecol 1989; 54: 66-70.

13. Martínez MA, Pinto E, ARroyo R. Incidencia de infección cervical por Chlamydia trachomatis y mycoplasma urogenitales en un consultorio de enfermedades de transmisión sexual. Rev Méd Chile 1986; 113: 118-21.

14. Ovalue A, Martínez A, Casals A, Yuhaniak R, Giglio M. Estudio clínico y microbiológico de la enfermedad inflamatoria pélvica aguda. Rev Chil Obstet Ginecol 1993; 58: 103-12.

15. Romero J, Prado V, Gaete V, Martínez D, Pizarro E. Infección por Chlamydia trachomatis en hombres chilenos asintomáticos y con uretritis. Utilidad de la onina de pnimer chomo. Rev Méd Chile 1997; 125: 1165-71.

16. González C, Cruz A, Bolívar N, Pizarro E. Epidemiología serológica de Chalmydia trachomatis en mujeres que asisten a un servicio de planificación familiar. Rev Chil Infect 1997; 15: 33-8.

17. Martínez MA, Ried I, Arias C, Napoltano C, Sandoval J, Molna R. Prevalencia de infección cervical por Chlamydia trachomatis en mujeres de la Región Metropolitana. Rev Méd Chile 2008; 136: 1294-300.

18. MuÑoz M, Romero P, MartínEz A. Infecciones de transmisión sexual: Experiencia en una unidad de ginecología pediátrica. Rev Chil Pediatr 2003; 74: 468-74.

19. ManRínQuez J, García M. Situación epidemiológica de gonorrea. El Vigía 24 Boletín de Vigilancia en Salud Pública de Chile 2007; 57-8. Disponible en http:// epi.minsal.cl/epi/html/elvigia/ elvigia.htm (Consultado 6 de septiembre de 2009).
20. Atkins D, Workowski K, Bauer H, Erbelding E, Geisler W, Hammersclag M ET AL. 2006 Sexually transmitted diseases guidelines. Disponible en www.cdc.gov (Consultado 6 de septiembre de 2009).

21. Boyadzhyan B, Yashina T, Yatabe JH, Patnaik M, HiLl CS. Comparison of the APTIMA CT and GC Assays with the APTIMA Combo 2 Assay, the Abbott LCx Assay, and Direct Fluorescent-Antibody and Culture Assays for Detection of Chlamydia trachomatis and Neisseria gonorrhoeae. J Clin Microbiol 2004; 42: 3089-93.

22. Johnson R, Newhall W, Papp J, Knapp J, Biack C, Gift T ET AL. Centers for Disease Control and Prevention Screening Tests To Detect Chlamydia trachomatis and Neisseria gonorrhoeae Infections - 2002». MMWR Recommendations and Reports 51(RR15);127. Disponible en http://www.aegis.com/PUBS/ mmwr/2002/RR5115-1.htm. (Revisado 12 de septiembre de 2009).

23. Low N, McCarthy A, Macleod J, Salisbury C, Campbell R, ROBERTS T ET AL. Epidemiological, social, diagnostic and economic evaluation of population screening for genital chlamydial infection. Health Technol Assess 2007; 11: 1-165.

24. Blas M, Canchinuaman F, Alva I, Hawes S. Pregnancy outcomes in women infected with Chlamydia trachomatis: a population-based cohort study in Washington State. Sex Transm Infect 2007; 83: 314-8.

25. GonZÁlzz C. Indicadores de Salud Chile 2007. p 10: Boletín 2007 Departamento de Estadística Ministerio de Salud, Chile. Disponible en www.redsalud.gov.cl (Revisado 12 de diciembre de 2008).

26. Valencia C, Prado V, Ríos M, Cruz M, Pilorget. Prevalencia de Chlamydia trachomatis en conjuntivitis neonatal determinada por técnicas de inmunofluoresencia y amplificación génica. Rev Méd Chile 2000; 128: 758-65.

27. Martínez M, Milán F, González C. Chlamydia trachomatis genotypes associated with pneumonia in Chilean infants. Scand J Infect Dis 2009; 41: 313-6.

28. Guimaraes E, Guimaraes M, Vieira M, Bontempo N, SeIXAS M, García MS ET AL. Lack of utility of risk score and gynecological examination for screening for sexually transmitted infections in sexually active adolescents. BMC Med 2009; 7: 8.

29. Hu D, Hook E, Gowde SJ. Screening for Chlamydia trachomatis in women 15 to 29 years of age: a costeffectiveness analysis. Ann Intern Med 2004; 141: 501-13.

30. Aravena A, Chamoro M, Camelio F, Candia E. $5^{a}$ Encuesta Nacional de Juventud, Chile 2006. Instituto Nacional de la Juventud, Gobierno de Chile. Disponible en www.injuv.gob.cl. (Consultado 6 de septiembre de 2009).

31. Eaton K, Kann L, Kinchen S, Shankuin S, Ross J, Hawkins J ET AL. Youth Risk Behavior Surveillance: United States, 2007. MMWR Surveill Summ 2008; 57: 1-131. 